\title{
Nonlinear conservation laws for the Schrödinger boundary value problems of second order
}

\author{
Ming Ren ${ }^{1 *}$, Shiwei Yun ${ }^{1}$ and Zhenping $\mathrm{Li}^{1}$
}

"Correspondence:
ren.ming@hotmail.com
'Department of Mathematics and
Physics, Luoyang Institute of
Science and Technology, Luoyang,
China

China

\begin{abstract}
In this paper, we apply a reliable combination of maximum modulus method with respect to the Schrödinger operator and Phragmén-Lindelöf method to investigate nonlinear conservation laws for the Schrödinger boundary value problems of second order. As an application, we prove the global existence to the solution for the Cauchy problem of the semilinear Schrödinger equation. The results reveal that this method is effective and simple.
\end{abstract}

Keywords: Conservation law; Schrödinger boundary value problem; Schrödinger equation

\section{Introduction}

In this article, we consider the following Schrödinger boundary value problems of second order (see [1-4, 12, 24, 29, 33, 38]):

$$
\begin{aligned}
& i f_{s}+\Delta f=-|f|^{p} f, \quad(t, s) \in \mathcal{R}^{n} \times[0, L), \\
& f(0, t)=f_{0}(t),
\end{aligned}
$$

where $i=\sqrt{-1}$,

$$
\Delta=\sum_{i=1}^{n} \frac{\partial^{2}}{\partial t_{i}^{2}}
$$

is the Laplace operator in $\mathcal{R}^{n}$,

$$
f(t, s): \mathcal{R}^{n} \times[0, L) \rightarrow \mathbb{C}
$$

denotes the complex valued function, $L$ is the maximum existence time, $n$ is the space dimension and $p$ satisfies the embedding condition

$$
\frac{4}{n}<p< \begin{cases}+\infty, & n=1,2 \\ \frac{4}{n-2}, & n>2 .\end{cases}
$$

(c) The Author(s) 2020. This article is licensed under a Creative Commons Attribution 4.0 International License, which permits use, sharing, adaptation, distribution and reproduction in any medium or format, as long as you give appropriate credit to the original author(s) and the source, provide a link to the Creative Commons licence, and indicate if changes were made. The images or other third party material in this article are included in the article's Creative Commons licence, unless indicated otherwise in a credit line to the material. If material is not included in the article's Creative Commons licence and your intended use is not permitted by statutory regulation or exceeds the permitted use, you will need to obtain permission directly from the copyright holder. To view a copy of this licence, visit http://creativecommons.org/licenses/by/4.0/. 
There has been a lot of interest in this Schrödinger equation, because of the significance in physics. Liu [24] investigated the stationary Schrödinger equation

$$
i f_{t}+\frac{1}{2} \Delta f+\frac{1}{2} \gamma \Delta^{2} f+|f|^{2 p} f=0
$$

where $\gamma \in \mathcal{R}, p \geq 1$, and the space dimension is no more than three. Problem (1.2) describes a stable soliton which is a wave pulse or wave beam, specially, there are solitons in magnetic materials for $p=1$ in $3 D$ space. Hu and Qiao [34] presented a numerical study on the axially symmetric fourth-order Schrödinger equation

$$
i \frac{\partial f}{\partial \xi}+\frac{1}{2} S \Delta_{\perp} f+\lambda \Delta_{\perp}^{2} f+\mu|f|^{2} f=0,
$$

where $S>0, \mu>0$,

$$
\Delta_{\perp}=\partial^{2} / \partial \rho^{2}+(1 / \rho) \partial / \partial \rho
$$

and $\xi, \rho$ are properly normalized cylindrical variables.

For $\lambda<0$, Eq. (1.3) plays a crucial role in the self-focusing, here the fourth derivative term in (1.3) may give rise to an oscillatory approach to the asymptotically homogeneous wave beam. Huang and Rui [22] analyzed the self-focusing and singularity formation in the mixed-dispersion nonlinear Schrödinger equation

$$
i f_{t}+\Delta f+\epsilon \Delta^{2} f+|f|^{2 p} f=0, \quad(t, s) \in \mathcal{R}^{n} \times[0, L)
$$

where $\epsilon= \pm 1, p \geq 1$, which occurs in propagation models for fiber arrays. The authors showed that the generic propagation dynamics for $\epsilon<0$ is focusing-defocusing oscillations. Qiao and Hou [33] considered the Schrödinger equation in dimensionless variables

$$
i f_{t}+D \Delta f+P \Delta^{2} f+B|f|^{2} f+K|f|^{4} f=0,
$$

where $D, P, B, K \in \mathcal{R}$ and $B K<0$. This equation was used for describing the dynamics of slowly varying wave packet envelope amplitude.

Given its mathematical interest, a lot of attention is paid to the existence and nonexistence of global solutions to the second-order boundary value problems related to the Schrödinger equation. Zhao [51] studied the equation

$$
i f_{t}+\Delta^{2} f+\beta \Delta f+\lambda|f|^{p-1} f=0, \quad(t, s) \in \mathcal{R}^{n} \times[0, L),
$$

where $\lambda, \beta \in \mathcal{R}, p \in\left(1,2^{\#}-1\right]$, and $2^{\#}=\frac{2 n}{n-4}$ is the energy critical exponent for the embedding from $H^{2}$ into Lebesgue's spaces.

On the other hand, we have the boundary value problem with respect to the Schrödinger operator corresponding to (1.5) given by (see $[6,9,10,15,17,26,40,41,44])$

$$
\begin{aligned}
& f \geq 0, \quad 0 \leq \omega \leq 1, \quad u(1-\omega)=0 \quad \text { in } P, \\
& \chi\left(t_{1}\right)\left(f_{t_{2}}+\omega\right)_{t_{2}}-\omega_{s}=0 \quad \text { in } P,
\end{aligned}
$$




$$
\begin{aligned}
& u=\phi \quad \text { on } \Sigma_{2}, \\
& \omega(\cdot, 0)=\omega_{0} \quad \text { in } J, \\
& \chi\left(t_{1}\right)\left(f_{t_{2}}+\omega\right) \cdot v=0 \quad \text { on } \Sigma_{1}, \\
& \chi\left(t_{1}\right)\left(f_{t_{2}}+\omega\right) \cdot v \leq 0 \quad \text { on } \Sigma_{4} .
\end{aligned}
$$

Regarding the existence of a solution of the problem related to the Schrödinger operator (1.1) we refer to [33] and [24], respectively, for the evolutionary dam problem with homogeneous coefficients and for a class of free boundary problem with respect to the Schrödinger operator in heterogeneous domain. The regularity of the solution of the problem [24] with respect to the Schrödinger operator was discussed in [34] (see also $[13,20,21,31,36])$, where it was proved that $\omega \in C^{0}\left([0, L] ; L^{p}(\beth)\right)$ for all $p \in[1,+\infty)$ in the class of free boundary problems with respect to the Schrödinger operator of types

$$
\begin{aligned}
& i f_{t}-\Delta^{2} f=-|f|^{2 p} u, \quad(t, s) \in \times \mathcal{R}^{n} \times[0, L), \\
& f(t, 0)=f_{0}(t)
\end{aligned}
$$

and that $f \in C^{0}\left([0, M] ; L^{p}(\beth)\right)$ for all $p \in[1,2]$ in the second-order class. More results as regards Schrödinger-type equations, wavelet analysis, distribution theory and calculus of variations were studied in previous work $[16,18,19,27,32,50]$. The semilinear elliptic equation on $\mathcal{R}^{n}$ was considered in [7]. The existence of infinitely many solutions to it under a variety of additional conditions was proved. Bound state solutions of sublinear Schrödinger equations with lack of compactness were studied in [8]. The existence of ground state solutions for nonlinear fractional Schrödinger equation was obtained in [11] by applying the minimization method with a constraint over a Pohožaev manifold. A diffusion model of Kirchhoff-type driven by a nonlocal integro-differential operator was considered in [48]. The existence of multiple solutions for the non-homogeneous fractional $p$-Laplacian equations of Schrödinger-Kirchhoff type was also considered in [45]. Boundary value problems driven by a combination of differential operators of different nature (such as (p,2)-equations) were studied in [30]. In 2019, Xue and Tang [49] established the existence of bound state solutions for a class of quasilinear Schrödinger equations whose nonlinear term is asymptotically linear in $\mathcal{R}^{n}$. After changing the variables, the quasilinear equation becomes a semilinear equation, whose respective associated functional is well defined in $H_{1}\left(\mathcal{R}^{n}\right)$. The proofs are based on the Pohozaev manifold and a linking theorem. As an application of the Phragmén-Lindelöf method related to a second-order boundary value problem with respect to the Schrödinger equation, in this paper we consider the conservation laws for a second-order boundary value problems related to the Schrödinger equation. As an application, we prove the global existence to the solution for the Cauchy problem of the semilinear Schrödinger equation. The results reveal that this method is effective and simple.

\section{Lemmas}

In this section, we obtain some lemmas which will be needed in the sequel. 
For the second-order boundary value problem (1.1) of the Schrödinger equation for $0<$ $\mathbf{E}\left(f_{0}\right)<d$, we have

$$
\Im(f)=\int_{\mathcal{R}^{n}}\left(|f|^{2}+|\nabla f|^{2}-\frac{n p}{2(p+2)}|f|^{p+2}\right) d t
$$

and

$$
\mathfrak{J}^{\prime \prime}(s)=8 \int_{\mathcal{R}^{n}}\left(|\nabla f|^{2}-\frac{n p}{2(p+2)}|f|^{p+2}\right) d t .
$$

Obviously, $\mathfrak{J}^{\prime \prime}(s)$ has a very similar structure with the Nehari functional $\mathfrak{I}(f)$, hence $\mathfrak{I}(f)<$ 0 can easily yield $\mathfrak{J}^{\prime \prime}(s)<0$ to prove the blowup of the solution.

But for the second-order boundary value problem (1.1) of the fourth-order semilinear Schrödinger equation, we do not have such luck. We shall derive in the main part of this paper that the corresponding $\mathcal{J}^{\prime \prime}(s)$ for the fourth-order semilinear Schrödinger equation is (see [37])

$$
\begin{aligned}
\mathcal{J}^{\prime \prime}(s)= & 8\left(4 \int_{\mathcal{R}^{n}}|\nabla(\Delta f)|^{2} d t+4 \int_{\mathcal{R}^{n}}|\Delta f|^{2} d t+\int_{\mathcal{R}^{n}}|\nabla f|^{2} d t\right) \\
& +4\left(-\frac{n p}{p+2} \int_{\mathcal{R}^{n}}|f|^{p+2} d t+(2 n+4) \operatorname{Re} \int_{\mathcal{R}^{n}}|f|^{p} f \Delta \bar{f} d t\right. \\
& \left.+4 \operatorname{Re} \int_{\mathcal{R}^{n}}|f|^{p} f x \cdot \nabla(\Delta \bar{f}) d t\right)
\end{aligned}
$$

by comparing it with

$$
\mathcal{I}(f)=\int_{\mathcal{R}^{n}}\left(|f|^{2}+|\nabla f|^{2}+|\Delta f|^{2}-\frac{n p}{2(p+2)}|f|^{p+2}\right) d t .
$$

We define the energy functional

$$
\mathfrak{E}(f)=\int_{\mathcal{R}^{n}}\left(\frac{1}{2}|\nabla f|^{2}-\frac{1}{p+2}|f|^{p+2}\right) d t,
$$

the auxiliary functionals

$$
\mathfrak{P}(f)=\int_{\mathcal{R}^{n}}\left(\frac{1}{2}|f|^{2}+\frac{1}{2}|\nabla f|^{2}-\frac{1}{p+2}|f|^{p+2}\right) d t
$$

and

$$
\Im(f)=\int_{\mathcal{R}^{n}}\left(|f|^{2}+|\nabla f|^{2}-\frac{n p}{2(p+2)}|f|^{p+2}\right) d t .
$$

For the above two functionals, $\mathfrak{P}(f)$ is composed of both mass and energy, and $\mathfrak{I}(f)$ can be considered as Nehari functional. Further we define the Hilbert space

$$
\mathfrak{H}=\left\{f \in H^{1}\left(\mathcal{R}^{n}\right): \int_{\mathcal{R}^{n}}|t|^{2}|f|^{2} d t<\infty\right\},
$$


the Nehari manifold

$$
\mathfrak{M}=\left\{f \in H^{1}\left(\mathcal{R}^{n}\right) \backslash\{0\}: \mathfrak{I}(f)=0\right\},
$$

the invariant manifolds

$$
\mathfrak{G}=\{f \in \mathfrak{H}: \mathfrak{P}(f)<\mathfrak{d}, \mathfrak{I}(f)>0\} \cup\{0\}
$$

and

$$
\mathfrak{B}=\{f \in \mathfrak{H}: \mathfrak{P}(f)<\mathfrak{d}, \mathfrak{I}(f)<0\},
$$

where $\mathfrak{d}=\inf _{f \in \mathfrak{M}} \mathfrak{P}(f)$.

\section{Lemma 2.1}

(i) For set $\mathfrak{G}$, we can obtain $\mathfrak{P}(f)>0$ by $\mathfrak{I}(f)>0$. So the set $\mathfrak{G}$ is equivalent to

$$
\mathfrak{G}^{\prime}=\{f \in \mathfrak{H} \mid 0<\mathfrak{P}(f)<\mathfrak{d}, \mathfrak{I}(f)>0\} \cup\{0\} .
$$

(ii) For set $\mathfrak{B}$, if $\mathfrak{P}(f) \leq 0$, we can get $\mathfrak{E}(f)<0$, which is a sufficient condition for finite time blowup; $c f$. [52]. Therefore, it is only necessary here to consider the case of $\mathfrak{E}(f)>0$, i.e., we only need

$$
\mathfrak{B}^{\prime}=\{f \in \mathfrak{H} \mid 0<\mathfrak{P}(f)<\mathfrak{d}, \mathfrak{I}(f)<0\} .
$$

The above remark is also applicable to sets $\mathcal{G}$ and $\mathcal{B}$ in Sect. 3.

For the second-order boundary value problem (1.1) of the second-order semilinear Schrödinger equation, we summarize some results established in [23, 35, 46, 47] as follows.

Lemma 2.2 Assume that $f_{0} \in \mathfrak{H}$ and $p$ satisfies the embedding condition [43]

$$
\frac{4}{n}<p< \begin{cases}+\infty, & n=1,2 \\ \frac{4}{n-2}, & n>2 .\end{cases}
$$

(i) There exist $L>0$ and a unique local solution $f(t, s)$ of problem (1.1) in $C\left(\left[0, L_{\max }\right] ; \mathfrak{H}\right)$. Moreover if

$$
L_{\max }=\sup \{L>0: u=f(t, s) \text { exists on }[0, L]\}<\infty
$$

then

$$
\lim _{t \rightarrow L_{\max }}\|f\|_{\mathfrak{H}}=\infty \quad \text { (blowup) }
$$

otherwise $L_{\max }=\infty$ (global existence). 
(ii) For the solution in (i),

$$
\begin{aligned}
& \int_{\mathcal{R}^{n}}|f(s)|^{2}=\int_{\mathcal{R}^{n}}\left|f_{0}\right|^{2} d t \quad \text { (mass conservation), } \\
& \mathcal{E}(f(s))=\mathfrak{E}\left(f_{0}\right) \quad \text { (energy conservation), } \\
& \mathfrak{P}(f(s)) \equiv \mathfrak{P}\left(f_{0}\right)
\end{aligned}
$$

(iii) $\mathfrak{d}>0, c f .[25,42]$.

(iv) If $f_{0} \in \mathfrak{G}$, then the solution of problem (1.1) is global.

(v) If $f_{0} \in \mathfrak{B}$, then the solution of problem (1.1) blows up in finite time.

In fact, although [28] proved the blowup solution by a variance of the argument in [39], there is no explicit computation of $\mathfrak{J}^{\prime \prime}(s)$. Now we give the specific computation of $\mathfrak{J}^{\prime \prime}(s)$ for the second-order boundary value problem (1.1).

Lemma 2.3 Assume that $f_{0} \in \mathfrak{B}, f(t, s) \in([0, L) ; \mathfrak{H})$ is the solution of $(1.1)$. Let $\mathfrak{J}(s)=$ $\int_{\mathcal{R}^{n}}|t|^{2}|f|^{2} d t$. Then

$$
\mathfrak{J}^{\prime \prime}(s)=8 \int_{\mathcal{R}^{n}}\left(|\nabla f|^{2}-\frac{n p}{2(p+2)}|f|^{p+2}\right) d t .
$$

Proof To prove the existence of the solution of (1.1), let $f$ be a solution of (1.1) for the value $\lambda$ of the parameter. Then, owing to (1.4), to the dominance of the principal eigenvalue of the operator $-\Delta^{2}$ in the domain $\mathcal{R}^{n} \times[0, L)$ under Dirichlet boundary conditions, to the facts that $\bar{f}_{t}$ is strongly positive in $\mathcal{R}^{n}$ and to the monotonicity of the principal eigenvalue with respect to the potential on the boundary conditions, we obtain

$$
\mathfrak{J}^{\prime}(s)=\int_{\mathcal{R}^{n}}|t|^{2}\left(u \bar{f}_{t}+\bar{f} f_{t}\right) d t
$$

which yields

$$
\mathfrak{J}^{\prime}(s)=\int_{\mathcal{R}^{n}}|t|^{2}\left(\overline{\bar{f}} f_{t}+\bar{f} f_{t}\right) d t=2 \operatorname{Re} \int_{\mathcal{R}^{n}}|t|^{2} \bar{f} f_{t} d t
$$

Multiplying both sides of (2.1) by $i$, we have

$$
f_{t}=i\left(\Delta f+|f|^{p} f\right)
$$

Substituting the above equation into (2.2) we have

$$
\begin{aligned}
\mathfrak{J}^{\prime}(s) & =2 \operatorname{Im} \int_{\mathcal{R}^{n}}|t|^{2}\left(f_{t} \Delta \bar{f}+f \Delta \bar{f}_{t}\right) d t \\
& =2 \operatorname{Im} \int_{\mathcal{R}^{n}}\left(|t|^{2} f_{t} \Delta \bar{f}+\Delta\left(|t|^{2} f\right) \bar{f}_{t}\right) d t \\
& =2 \operatorname{Im} \int_{\mathcal{R}^{n}}\left(|t|^{2} f_{t} \Delta \bar{f}+\bar{f}_{t} \sum_{i=1}^{n} \frac{\partial^{2}}{\partial t_{i}^{2}}\left(|t|^{2} f\right)\right) d t
\end{aligned}
$$




$$
\begin{aligned}
& =2 \operatorname{Im} \int_{\mathcal{R}^{n}}\left(|t|^{2} f_{t} \Delta \bar{f}+\bar{f}_{t} \sum_{i=1}^{n} \frac{\partial}{\partial t_{i}}\left(|t|^{2} \frac{\partial f}{\partial t_{i}}+2 t_{i} u\right)\right) d t \\
& =2 \operatorname{Im} \int_{\mathcal{R}^{n}}\left(|t|^{2} f_{t} \Delta \bar{f}+\bar{f}_{t}\left(2 n f+4 \sum_{i=1}^{n} t_{i} \cdot \frac{\partial f}{\partial t_{i}}+|t|^{2} \sum_{i=1}^{n} \frac{\partial^{2} u}{\partial t_{i}^{2}}\right)\right) d t \\
& =2 \operatorname{Im} \int_{\mathcal{R}^{n}}\left(|t|^{2} f_{t} \Delta \bar{f}+\bar{f}_{t}\left(2 n f+4 x \cdot \nabla f+|t|^{2} \Delta f\right)\right) d t \\
& =2 \operatorname{Im} \int_{\mathcal{R}^{n}}\left(|t|^{2} f_{t} \Delta \bar{f}+\overline{|t|^{2} f_{t} \Delta \bar{f}}+\bar{f}_{t}(2 n f+4 x \cdot \nabla f)\right) d t \\
& =4 \operatorname{Im} \int_{\mathcal{R}^{n}}(N u+2 x \cdot \nabla f) \bar{f}_{t} d t
\end{aligned}
$$

and

$$
\begin{aligned}
\mathfrak{J}^{\prime \prime}(s) & =2 \operatorname{Re} \int_{\mathcal{R}^{n}} i|t|^{2} \bar{f}\left(\Delta f+|f|^{p} f\right) d t \\
& =-2 \operatorname{Im} \int_{\mathcal{R}^{n}}|t|^{2} \bar{f}\left(\Delta f+|f|^{p} f\right) d t \\
& =-2 \operatorname{Im} \int_{\mathcal{R}^{n}}|t|^{2}\left(\bar{f} \Delta f+|f|^{p+2}\right) d t \\
& =-2 \operatorname{Im} \int_{\mathcal{R}^{n}}|t|^{2} \bar{f} \Delta f d t \\
& =2 \operatorname{Im} \int_{\mathcal{R}^{n}}|t|^{2} f \Delta \bar{f} d t .
\end{aligned}
$$

It follows from (2.1) that

$$
\bar{f}_{t}=-i\left(\Delta \bar{f}+|f|^{p} \bar{f}\right) .
$$

Substituting the above equation into (2.3), we can get

$$
\begin{aligned}
\mathfrak{J}^{\prime \prime}(s) & =-4 \operatorname{Im} \int_{\mathcal{R}^{n}} i(n f+2 x \cdot \nabla f)\left(\Delta \bar{f}+|f|^{p} \bar{f}\right) d t \\
& =-4 \operatorname{Re} \int_{\mathcal{R}^{n}}(n f+2 x \cdot \nabla f)\left(\Delta \bar{f}+|f|^{p} \bar{f}\right) d t \\
& =-4\left(\operatorname{Re} \int_{\mathcal{R}^{n}}(n f+2 x \cdot \nabla f) \Delta \bar{f} d t+\operatorname{Re} \int_{\mathcal{R}^{n}}(n f+2 x \cdot \nabla f)|f|^{p} \bar{f} d t\right) \\
& =-4\left(\mathfrak{I}_{1}+\mathfrak{I}_{2}\right),
\end{aligned}
$$

where

$$
\Im_{1}:=\operatorname{Re} \int_{\mathcal{R}^{n}}(n f+2 x \cdot \nabla f) \Delta \bar{f} d t
$$

and

$$
\mathfrak{I}_{2}:=\operatorname{Re} \int_{\mathcal{R}^{n}}(n f+2 x \cdot \nabla f)|f|^{p} \bar{f} d t .
$$


Rene et al. Boundary Value Problems

(2020) 2020:1

Page 8 of 14

For $\mathfrak{I}_{1}$ and $\mathfrak{I}_{2}$, we have

$$
\begin{aligned}
\mathfrak{I}_{1} & \left.=n \int_{\mathcal{R}^{n}}|f|^{p+2} d t+\operatorname{Re} \int_{\mathcal{R}^{n}} x \cdot\left(|f|^{p} \bar{f} \nabla f+u \nabla \bar{f}\right)\right) d t \\
& =n \int_{\mathcal{R}^{n}}|f|^{p+2} d t+\operatorname{Re} \int_{\mathcal{R}^{n}} x \cdot\left(|f|^{p} \nabla(u \bar{f})\right) d t \\
& =n \int_{\mathcal{R}^{n}}|f|^{p+2} d t+\operatorname{Re} \int_{\mathcal{R}^{n}} x \cdot\left(\left(|f|^{2}\right)^{p / 2} \nabla|f|^{2}\right) d t \\
& =n \int_{\mathcal{R}^{n}}|f|^{p+2} d t+\frac{2}{p+2} \operatorname{Re} \int_{\mathcal{R}^{n}} x \cdot \nabla\left(|f|^{2}\right)^{\frac{p+2}{2}} d t \\
& =n \int_{\mathcal{R}^{n}}|f|^{p+2} d t-\frac{2 n}{p+2} \operatorname{Re} \int_{\mathcal{R}^{n}}|f|^{p+2} d t \\
& =\frac{n p}{p+2} \operatorname{Re} \int_{\mathcal{R}^{n}}|f|^{p+2} d t
\end{aligned}
$$

and

$$
\begin{aligned}
\mathfrak{I}_{2}= & N \operatorname{Re} \int_{\mathcal{R}^{n}} f \Delta \bar{f} d t+2 \operatorname{Re} \int_{\mathcal{R}^{n}}(t \cdot \nabla f) \Delta \bar{f} d t \\
= & -n \int_{\mathcal{R}^{n}}|\nabla f|^{2} d t-2 \operatorname{Re} \int_{\mathcal{R}^{n}} \nabla(t \cdot \nabla f) \nabla \bar{f} d t \\
= & -n \int_{\mathcal{R}^{n}}|\nabla f|^{2} d t-2 \operatorname{Re} \int_{\mathcal{R}^{n}} \sum_{i=1}^{n} \frac{\partial}{\partial t_{i}}\left(\sum_{j=1}^{n} t_{j} \frac{\partial f}{\partial t_{j}}\right) \frac{\partial \bar{f}}{\partial t_{i}} d t \\
= & -n \int_{\mathcal{R}^{n}}|\nabla f|^{2} d t-2 \operatorname{Re} \int_{\mathcal{R}^{n}} \sum_{i=1}^{n} \sum_{j=1}^{n} \frac{\partial}{\partial t_{i}}\left(t_{j} \frac{\partial f}{\partial t_{j}}\right) \frac{\partial \bar{f}}{\partial t_{i}} d t \\
= & -n \int_{\mathcal{R}^{n}}|\nabla f|^{2} d t-2 \operatorname{Re} \int_{\mathcal{R}^{n}} \sum_{i=1}^{n} \frac{\partial f}{\partial t_{i}} \frac{\partial \bar{f}}{\partial t_{i}} d t \\
& -2 \operatorname{Re} \int_{\mathcal{R}^{n}} \sum_{i=1}^{n} \sum_{j=1}^{n} t_{j} \frac{\partial^{2} u}{\partial t_{i} \partial t_{j}} \frac{\partial \bar{f}}{\partial t_{i}} d t \\
= & -n \int_{\mathcal{R}^{n}}|\nabla f|^{2} d t-2 \int_{\mathcal{R}^{n}}|\nabla f|^{2} d t \\
& -\operatorname{Re} \int_{\mathcal{R}^{n}} \sum_{i=1}^{n} \sum_{j=1}^{n} t_{j}\left(\frac{\partial^{2} u}{\partial t_{i} \partial t_{j}} \frac{\partial \bar{f}}{\partial t_{i}}+\frac{\partial^{2} \bar{f}}{\partial t_{i} \partial t_{j}} \frac{\partial f}{\partial t_{i}}\right) d t \\
= & -n \int_{\mathcal{R}^{n}}|\nabla f|^{2} d t-2 \int_{\mathcal{R}^{n}}|\nabla f|^{2} d t-\operatorname{Re} \int_{\mathcal{R}^{n}} \sum_{i=1}^{n} \sum_{j=1}^{n} t_{j} \frac{\partial}{\partial t_{j}}\left(\frac{\partial f}{\partial t_{i}} \frac{\partial \bar{f}}{\partial t_{i}}\right) d t \\
= & -n \int_{\mathcal{R}^{n}}|\nabla f|^{2} d t-2 \int_{\mathcal{R}^{n}}|\nabla f|^{2} d t-\operatorname{Re} \int_{\mathcal{R}^{n}} x \cdot \nabla|\nabla f|^{2} d t \\
= & -n \int_{\mathcal{R}^{n}}|\nabla f|^{2} d t-2 \int_{\mathcal{R}^{n}}|\nabla f|^{2} d t+n \int_{\mathcal{R}^{n}}|\nabla f|^{2} d t \\
& -2 .
\end{aligned}
$$


Substituting the above equation and (2.6) into (2.5), finally we derive

$$
\begin{aligned}
\mathfrak{J}^{\prime \prime}(s) & =-4\left(-2 \int_{\mathcal{R}^{n}}|\nabla f|^{2} d t+\frac{n p}{p+2} \operatorname{Re} \int_{\mathcal{R}^{n}}|f|^{p+2} d t\right) \\
& =8 \int_{\mathcal{R}^{n}}\left(|\nabla f|^{2}-\frac{n p}{2(p+2)}|f|^{p+2}\right) d t .
\end{aligned}
$$

Then the proof is complete.

\section{Main result}

In this section, we shall state and prove our main result.

Theorem 3.1 (Conservation laws) Let $f_{0} \in \mathcal{H}^{2}$ and $f \in\left([0, L) ; \mathcal{H}^{2}\right)$ be the unique solution of problem (1.1). Then

$$
\begin{aligned}
& \int_{\mathcal{R}^{n}}|f(s)|^{2}=\int_{\mathcal{R}^{n}}\left|f_{0}\right|^{2} d t \quad \text { (mass conservation) }, \\
& \mathcal{E}(f(s))=\mathcal{E}\left(f_{0}\right) \quad(\text { energy conservation }), \\
& \mathcal{P}(f(s)) \equiv \mathcal{P}\left(f_{0}\right) .
\end{aligned}
$$

Proof It follows that

$$
\begin{aligned}
\frac{d}{d t}\left(\int_{\mathcal{R}^{n}}|f|^{2} d t\right) & =\frac{d}{d t}\left(\int_{\mathcal{R}^{n}} u \bar{f} d t\right) \\
& =\int_{\mathcal{R}^{n}}\left(u \bar{f}_{t}+f_{t} \bar{f}\right) d t \\
& =\int_{\mathcal{R}^{n}}\left(\overline{f_{t}} \bar{f}+f_{t} \bar{f}\right) d t \\
& =2 \operatorname{Re} \int_{\mathcal{R}^{n}} \bar{f} f_{t} d t
\end{aligned}
$$

from the definitions of the energy functional $\mathcal{E}(f(s))$ and $\mathcal{P}(f(s))$, which yields

$$
\bar{f} f_{t}=i\left(\bar{f} \Delta f-\bar{f} \Delta^{2} f+|f|^{p+2}\right) .
$$

It follows from (3.4) and (3.5) that

$$
\begin{aligned}
\frac{d}{d t}\left(\int_{\mathcal{R}^{n}}|f|^{2} d t\right) & =2 \operatorname{Re} \int_{\mathcal{R}^{n}} i\left(\bar{f} \Delta f-\bar{f} \Delta^{2} u+|f|^{p+2}\right) d t \\
& =-2 \operatorname{Im} \int_{\mathcal{R}^{n}}\left(\bar{f} \Delta f-\bar{f} \Delta^{2} u+|f|^{p+2}\right) d t \\
& =2 \operatorname{Im} \int_{\mathcal{R}^{n}}\left(|\nabla f|^{2}+|\Delta f|^{2}-|f|^{p+2}\right) d t=0 .
\end{aligned}
$$

So (3.1) holds. 
Then we prove the energy conservation as follows:

$$
\begin{aligned}
\frac{d}{d t}(\mathcal{E}(f(s)))= & \frac{d}{d t}\left(\int_{\mathcal{R}^{n}}\left(\frac{1}{2}|\nabla f|^{2}+\frac{1}{2}|\Delta f|^{2}-\frac{1}{p+2}|f|^{p+2}\right) d t\right) \\
= & \frac{d}{d t}\left(\int_{\mathcal{R}^{n}}\left(\frac{1}{2} \nabla f \cdot \nabla \bar{f}+\frac{1}{2} \Delta f \Delta \bar{f}-\frac{1}{p+2}(u \bar{f})^{\frac{p+2}{2}}\right) d t\right) \\
= & \int_{\mathcal{R}^{n}}\left(\frac{1}{2}\left(\nabla f_{t} \cdot \nabla \bar{f}+\nabla f \cdot \nabla \bar{f}_{t}\right)+\frac{1}{2}\left(\Delta f_{t} \Delta \bar{f}+\Delta f \Delta \bar{f}_{t}\right)\right. \\
& \left.-\frac{1}{2}(u \bar{f})^{p / 2}\left(\bar{f}_{t} \bar{f}+u \bar{f}_{t}\right)\right) d t,
\end{aligned}
$$

which yields

$$
\begin{aligned}
\frac{d}{d t}(\mathcal{E}(f(s)))= & \frac{d}{d t}\left(\int_{\mathcal{R}^{n}}\left(\frac{1}{2}|\nabla f|^{2}+\frac{1}{2}|\Delta f|^{2}-\frac{1}{p+2}|f|^{p+2}\right) d t\right) \\
= & \frac{d}{d t}\left(\int_{\mathcal{R}^{n}}\left(\frac{1}{2} \nabla f \cdot \nabla \bar{f}+\frac{1}{2} \Delta f \Delta \bar{f}-\frac{1}{p+2}(u \bar{f})^{\frac{p+2}{2}}\right) d t\right) \\
= & \int_{\mathcal{R}^{n}}\left(\frac{1}{2}\left(\nabla f_{t} \cdot \nabla \bar{f}+\nabla f \cdot \nabla \overline{f_{t}}\right)+\frac{1}{2}\left(\Delta f_{t} \Delta \bar{f}+\Delta f \Delta \bar{f}_{t}\right)\right. \\
& \left.-\frac{1}{2}(u \bar{f})^{p / 2}\left(f_{t} \bar{f}+u \bar{f}_{t}\right)\right) d t \\
= & \int_{\mathcal{R}^{n}}\left(\frac{1}{2}\left(\nabla f_{t} \cdot \nabla \bar{f}+\overline{\nabla \bar{f} \cdot \nabla f_{t}}\right)+\frac{1}{2}\left(\Delta f_{t} \Delta \bar{u}+\overline{\Delta \bar{u} \Delta f_{t}}\right)\right. \\
& \left.-\frac{1}{2}(u \bar{f})^{p / 2}\left(f_{t} \bar{f}+\overline{u \bar{f}_{t}}\right)\right) d t \\
= & \operatorname{Re} \int_{\mathcal{R}^{n}}\left(\left(\nabla f_{t} \cdot \nabla \bar{f}\right)+\left(\Delta f_{t} \Delta \bar{f}\right)-(u \bar{f})^{p / 2}\left(f_{t} \bar{f}\right)\right) d t \\
= & -\operatorname{Re} \int_{\mathcal{R}^{n}}\left(f_{t} \Delta \bar{f}-f_{t} \Delta^{2} \bar{f}+|f|^{p} \bar{f} f_{t}\right) d t \\
= & -\operatorname{Re} \int_{\mathcal{R}^{n}} f_{t}\left(\Delta \bar{f}-\Delta^{2} \bar{f}+|f|^{\bar{f}}\right) d t .
\end{aligned}
$$

So

$$
i\left|f_{t}\right|^{2}=-\bar{f}_{t}\left(\Delta f-\Delta^{2} u+|f|^{p} f\right) .
$$

Then substituting the above equation into (3.6) gives

$$
\frac{d}{d t}(\mathcal{E}(f(s)))=\operatorname{Re} \int_{\mathcal{R}^{n}} i\left|f_{t}\right|^{2} d t=-\operatorname{Im} \int_{\mathcal{R}^{n}}\left|f_{t}\right|^{2} d t=0,
$$

thus (3.2) holds.

Finally, we obtain (3.3) from (3.1) and (3.2).

\section{An application}

As a crucial application, we prove the global existence to the solution for the Cauchy problem of the semilinear Schrödinger equation. 
As shown in $[5,14]$, the negative initial energy $\left(\mathcal{E}\left(f_{0}\right)<0\right)$ is currently the sufficient condition for blowup of the Cauchy problem (1.1), i.e., in this case, it is impossible to divide the initial condition to obtain the sharp condition of global existence and blowup in the frame of the variational method. Therefore, we only consider the case of $0<\mathcal{E}\left(f_{0}\right)<d$ and try to build a similar result to the second-order semilinear Schrödinger equation. First we need to verify $d>0$.

Lemma 4.1 The depth of the potential well is positive, i.e., $d>0$.

Proof It follows from the Sobolev embedding inequalities that

$$
\begin{aligned}
\int_{\mathcal{R}^{n}}\left(|\nabla f|^{2}+|f|^{2}\right) d t & \leq \int_{\mathcal{R}^{n}}\left(|\nabla f|^{2}+|f|^{2}+|\Delta f|^{2}\right) d t \\
& =\frac{n p}{2(p+2)} \int_{\mathcal{R}^{n}}|f|^{p+2} d t \\
& \leq \frac{n p}{2(p+2)}\left(\int_{\mathcal{R}^{n}} c\left(|\nabla f|^{2}+|f|^{2}\right) d t\right)^{\frac{p+2}{2}}
\end{aligned}
$$

for any $f \in M$.

Put

$$
C=\left(\frac{2(p+2)}{n p}\right)^{2 / p} c^{-\frac{p+2}{p}}
$$

Then

$$
0<C \leq \int_{\mathcal{R}^{n}}\left(|\nabla f|^{2}+|f|^{2}\right) d t
$$

It follows from (4.1) and the definition of $\mathcal{P}(f)$ that

$$
\begin{aligned}
\mathcal{P}(f)= & \int_{\mathcal{R}^{n}}\left(\frac{1}{2}|f|^{2}+\frac{1}{2}|\nabla f|^{2}+\frac{1}{2}|\Delta f|^{2}-\frac{1}{p+2}|f|^{p+2}\right) d t \\
= & \left(\frac{1}{2}-\frac{1}{p+2} \cdot \frac{2(p+2)}{n p}\right) \int_{\mathcal{R}^{n}}\left(|f|^{2}+|\nabla f|^{2}+|\Delta f|^{2}\right) d t \\
& +\frac{1}{p+2} \cdot \frac{2(p+2)}{n p} \int_{\mathcal{R}^{n}}\left(|f|^{2}+|\nabla f|^{2}+|\Delta f|^{2}-\frac{n p}{2(p+2)}|f|^{p+2}\right) d t \\
= & \frac{n p-2}{2 n p} \int_{\mathcal{R}^{n}}\left(|f|^{2}+|\nabla f|^{2}+|\Delta f|^{2}\right) d t \\
\geq & \frac{n p-2}{2 n p} \int_{\mathcal{R}^{n}}\left(|\nabla f|^{2}+|f|^{2}\right) d t \\
\geq & C>0 .
\end{aligned}
$$

Finally, we obtain the desired result.

Theorem 4.1 Let $f_{0} \in \mathcal{G}$. Then the solution $f(t, s)$ of the initial value problem (1.1) be global, i.e., the maximum existence time $L=\infty$. 
Proof It follows from Lemma 4.1 that

$$
\begin{aligned}
d>\mathcal{P}(f)= & \int_{\mathcal{R}^{n}}\left(\frac{1}{2}|f|^{2}+\frac{1}{2}|\nabla f|^{2}+\frac{1}{2}|\Delta f|^{2}-\frac{1}{p+2}|f|^{p+2}\right) d t \\
= & \left(\frac{1}{2}-\frac{1}{p+2} \cdot \frac{2(p+2)}{n p}\right) \int_{\mathcal{R}^{n}}\left(|f|^{2}+|\nabla f|^{2}+|\Delta f|^{2}\right) d t \\
& +\frac{1}{p+2} \cdot \frac{2(p+2)}{n p} \int_{\mathcal{R}^{n}}\left(|f|^{2}+|\nabla f|^{2}+|\Delta f|^{2}-\frac{n p}{2(p+2)}|f|^{p+2}\right) d t \\
\geq & \frac{n p-2}{2 n p} \int_{\mathcal{R}^{n}}\left(|f|^{2}+|\nabla f|^{2}+|\Delta f|^{2}\right) d t
\end{aligned}
$$

for any $t \in[0, L)$, which yields

$$
\int_{\mathcal{R}^{n}}\left(|\nabla f|^{2}+|f|^{2}+|\Delta f|^{2}\right) d t \leq \frac{2 d n p}{n p-2}
$$

Then according to Theorem 3.1, the existence time of a local solution of (1.1) can be extended to infinity, thus the solution of the problem (1.1) is global.

\section{Conclusions}

In this paper, we applied a reliable combination of maximum modulus method with respect to the Schrödinger operator and Phragmén-Lindelöf method to investigate conservation laws for a second-order boundary value problems related to the Schrödinger equation. As an application, we prove the global existence to the solution for the Cauchy problem of the semilinear Schrödinger equation. The results reveal that this method is effective and simple.

\section{Acknowledgements}

Not applicable.

Funding

Not applicable.

\section{Abbreviations}

Not applicable.

Availability of data and materials

Not applicable.

Competing interests

The authors declare that there are no competing interests.

Authors' contributions

The authors completed the paper and approved the final manuscript.

\section{Publisher's Note}

Springer Nature remains neutral with regard to jurisdictional claims in published maps and institutional affiliations.

Received: 5 September 2019 Accepted: 17 December 2019 Published online: 03 January 2020

\section{References}

1. Acerbi, E., Fusco, N.: A transmission problem in the calculus of variations. Calc. Var. Partial Differ. Equ. 2, 1-16 (1994)

2. Amann, H.: Nonlinear elliptic equations with nonlinear boundary conditions. In: Eckhaus, W. (ed.) New Developments in Differential Equations. Math Studies, vol. 21, pp. 43-63. North-Holland, Amsterdam (1976)

3. Anderson, R.M., May, R.M.: Population Biology of Infectious Diseases. Springer, Berlin (1982)

4. Anderson, R.M., May, R.M., Anderson, B.: Infectious Diseases of Humans: Dynamics and Control. Oxford University Press, Oxford (1992) 
5. Andreu, F., Mazón, J.M., Toledo, J., Rossi, J.D.: Porous medium equation with absorption and a nonlinear boundary condition. Nonlinear Anal. 49, 541-563 (1992)

6. Assing, S., Manthey, R.: The behavior of solutions of stochastic differential inequalities. Probab. Theory Relat. Fields 103, 493-514 (1995)

7. Bahrouni, A., Ounaies, H., Radulescu, V.D.: Infinitely many solutions for a class of sublinear Schrödinger equations with indefinite potentials. Proc. R. Soc. Edinb., Sect. A 145(3), 445-465 (2015)

8. Bahrouni, A., Ounaies, H., Radulescu, V.D.: Bound state solutions of sublinear Schrödinger equations with lack of compactness. Rev. R. Acad. Cienc. Exactas Fís. Nat., Ser. A Mat. 113(2), 1191-1210 (2019)

9. Carrillo, J.: On the uniqueness of the solution of the evolution Dam problem. Nonlinear Anal., Theory Methods Appl. 22, 573-607 (1994)

10. Carrillo, J., Gilardi, G.: La vitesse de propagation dans le problème de la digue. Ann. Fac. Sci. Toulouse 11(3), 7-28 (1990)

11. Chen, J., Gao, Z:: Ground state solutions for fractional Schrödinger equation with variable potential and Berestycki-Lions type nonlinearity. Bound. Value Probl. 2019, 148 (2019)

12. Chen, X.F., Friedman, A.: A free boundary problem arising in a model of wound healing. SIAM J. Math. Anal. 32, 778-800 (1998)

13. Cheng, X., Wu, F: Several conservative compact schemes for a class of nonlinear Schrödinger equations with wave operator. Bound. Value Probl. 2018, 40 (2018)

14. Chipot, M., Fila, M., Quittner, P.: Stationary solutions, blow up and convergence to stationary solutions for semilinear parabolic equations with nonlinear boundary conditions. Acta Math. Univ. Comen. 60, 35-103 (1991)

15. Dibenedetto, E., Friedam, A.: Periodic behaviour for the evolutionary dam problem and related free boundary problems. Commun. Partial Differ. Equ. 11, 1297-1377 (1986)

16. Diekmann, O.: Run for your life. A note on the asymptotic speed of propagation of an epidemic. J. Differ. Equ. 33, 58-73 (1979)

17. Gilardi, G.: A new approach to evolution free boundary problems. Commun. Partial Differ. Equ. 4, 1099-1123 (1979)

18. Gilbarg, D., Trudinger, N.S.: Elliptic Partial Differential Equations of Second Order. Springer, New York (1983)

19. Guariglia, E., Silvestrov, S.: Fractional-Wavelet Analysis of Positive Definite Distributions and Wavelets on $\mathcal{D}^{\prime}(C)$. Engineering Mathematics. II. Springer Proc. Math. Stat., vol. 179, pp. 337-353. Springer, Cham (2016)

20. Guliyev, V.S., Guliyev, R.V., Omarova, M., Ragusa, M.A.: Schrödinger type operators on local generalized Morrey spaces related to certain nonnegative potentials. Discrete Contin. Dyn. Syst., Ser. B 25(2), 671-690 (2020)

21. Hao, Y., Li, P., Zhao, K.: Regularities of semigroups, Carleson measures and the characterizations of BMO-type spaces associated with generalized Schrödinger operators. Banach J. Math. Anal. 13(1), 1-25 (2019)

22. Huang, X., Rui, W.: Different kinds of exact solutions of fractional generalized Bagley-Torvik equation and their dynamical properties. J. Yunnan Univ. Nat. Sci. 40, 12-21 (2018)

23. Kermack, W.O., M'Kendrick, A.D.: A contribution to the mathematical theory of epidemics. Proc. R. Soc. Lond. A 115, 700-721 (1927)

24. Liu, Z.: Existence results for the general Schrödinger equations with a superlinear Neumann boundary value problem. Bound. Value Probl. 2019, 61 (2019)

25. López-Gómez, J., Marquez, V., Wolanski, N.: Blow up results and localization of blow up points for the heat equations with a nonlinear boundary conditions. J. Differ. Equ. 92, 384-401 (1991)

26. Lyaghfouri, A.: The evolution dam problem for nonlinear Darcy's law and Dirichlet boundary conditions. Port. Math. 56(1), 1-38 (1999)

27. Mikhaylov, A., Mikhaylov, V: The boundary control method and de Branges spaces. Schrödinger equation, Dirac system and discrete Schrödinger operator. J. Math. Anal. Appl. 460(2), 927-953 (2018)

28. Mozer, J.: A new proof of De Giorgi's theorem concerning the regularity problem for elliptic differential equations. Commun. Pure Appl. Math. 13(3), 457-468 (1960)

29. Murray, J.D.: Mathematical Biology. II. Spatial Models and Biomedical Applications, 3rd edn. Interdisciplinary Applied Mathematics, vol. 18. Springer, New York (2003)

30. Papageorgiou, N.S., Radulescu, V.D., Repovs, D.D.: On a class of parametric $(p, 2)$-equations. Appl. Math. Optim. 75(2), 193-228 (2017)

31. Peng, W., Tian, S., Zhang, T.: On the breather waves, rogue waves and solitary waves to a generalized (2 + 1)-dimensional Caudrey-Dodd-Gibbon-Kotera-Sawada equation. Filomat 32(14), 4959-4969 (2018)

32. Polidoro, S., Ragusa, M.A.: On some Schrödinger type equations. In: More Progresses in Analysis. World Scientific, Singapore (2009)

33. Qiao, Y., Hou, G.: Green matrix of eigenvalue problem and completeness of eigenfunction systems for a Hamiltonian operator derived from the wave equations. J. Inn. Mong. Univ. Nat. Sci. 49, 113-119 (2018)

34. Hu, B., Qiao, Y.: Existence of solution for a first-order ordinary differential equation under anti-periodic boundary value conditions with impulsive integral condition. J. Syst. Sci. Math. Sci. 33, 962-975 (2013)

35. Rabinowitz, P.H.: Some global results for nonlinear eigenvalue problems. J. Funct. Anal. 7, 487-513 (1971)

36. Ragusa, M.: Cauchy-Dirichlet problem associated to divergence form parabolic equations. Commun. Contemp. Math. 6(3), 377-393 (2004)

37. Rass, L., Radcliffe, J.: Spatial Deterministic Epidemics. Am. Math. Soc., Providence (2003)

38. Rawashdeh, Z: A free boundary problem for a predator-prey model. Nonlinearity 20, 1883-1892 (2001)

39. Rossi, J.D.: Elliptic problems with nonlinear boundary conditions and the Sobolev trace theorem. In: Chipot, M. Quittner, P. (eds.) Handbook of Differential Equations: Stationary Partial Differential Equations, vol. 2, Chap. 5 , pp. 311-406. Elsevier, Amsterdam (1995)

40. Rossi, J.D.: The blow-up rate for a semilinear parabolic equation with a nonlinear boundary condition. Acta Math. Univ. Comen. 67, 343-350 (1998)

41. Sattinger, D.: Topics in Stability and Bifurcation Theory. Lectures Notes in Mathematics, vol. 309. Springer, Berlin (1973)

42. Serrin, J.: Local behavior of solutions of quasilinear elliptic equations. Acta Math. 111, 247-302 (1964)

43. Simms, D:: Notes on the results of lower bounds for a class of harmonic functions in the half space. Filomat 32(8), 2981-2993 (2018) 
44. Stein, E.M.: Singular Integrals and Differentiability Properties of Functions. Princeton University Press, Princeton (1970)

45. Torres, C.: Multiplicity result for non-homogeneous fractional Schrodinger-Kirchhoff-type equations in $R^{n}$. Adv. Nonlinear Anal. 7(3), 247-257 (2018)

46. Walter, W: On existence and nonexistence in the large of solutions of parabolic differential equations with a nonlinear boundary condition. SIAM J. Math. Anal. 6(1), 85-90 (1975)

47. Willem, M.: Minimax Theorems. Birkhäuser, Boston (1996)

48. Xiang, M., Radulescu, V.D., Zhang, B.: Nonlocal Kirchhoff diffusion problems: local existence and blow-up of solutions. Nonlinearity 31(7), 3228-3250 (2018)

49. Xue, Y.F., Tang, C.: Existence of a bound state solution for quasilinear Schrödinger equations. Adv. Nonlinear Anal. 8(1), 323-338 (2019)

50. Zhang, X., Liu, D., Yan, Z., Zhao, G., Yuan, Y.: Schrödinger-type identity for Schrödinger free boundary problems. Bound. Value Probl. 2018, 135 (2018)

51. Zhao, L.: A Schrödinger-type algorithm for solving the Schrödinger equations via Phragmén-Lindelöf inequalities. J. Inequal. Appl. 2019, 144 (2019)

52. Zhikov, V.V.: Averaging of functionals of the calculus of variations and elasticity theory. Izv. Akad. Nauk SSSR, Ser. Mat. 50(4), 675-711 (1986)

\section{Submit your manuscript to a SpringerOpen ${ }^{\circ}$ journal and benefit from:}

- Convenient online submission

- Rigorous peer review

- Open access: articles freely available online

- High visibility within the field

- Retaining the copyright to your article

Submit your next manuscript at $\boldsymbol{\nabla}$ springeropen.com 